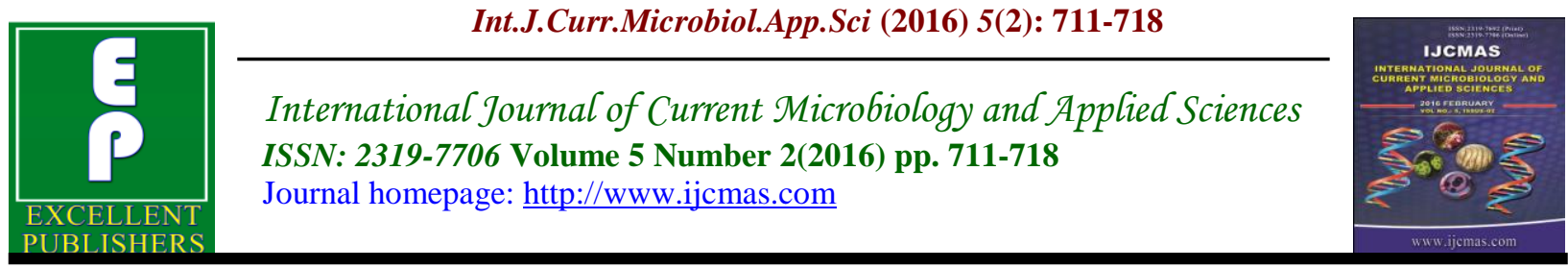

Original Research Article

doi: http://dx.doi.org/10.20546/ijcmas.2016.502.079

\title{
Lactobacilli and Obesity
}

\author{
Akshita Ramani $^{1}$, S. Sangeetha ${ }^{2^{*}}$ and Y. K. Harshika ${ }^{3}$ \\ ${ }^{1}$ III MBBS Student, Rajarajeswari Medical College\& Hospital, Bangalore, India \\ ${ }^{2}$ Department of Microbiology, Rajarajeswari Medical College\& Hospital, Bangalore, India \\ ${ }^{3}$ Final Year Postgraduate, (M.D.) Microbiology and Hospital, Rajarajeswari Medical College, \\ Bangalore, India \\ *Corresponding author
}

Keywords

Lactobacilli

estimation, MRS

Agar, Obesity

MeSH Terms:

Lactobacilli,

Obesity,

Correlation.

Article Info

Accepted:

28 January 2016

Available Online:

10, February 2016

\section{A B S T R A C T}

Obesity as a disease contributes to over 2.8 million deaths annually worldwide. Studies, the world over are attributing obesity as both cause and effect of changes in intestinal microflora. A culture based study to 1) Observe alterations, in the number of Lactobacilli in obese individuals. 2) Substantiate the role of gut microflora in obesity. A cross sectional study with two groups: 20 Obese $(\mathrm{O})$ and 20 as Controls $(\mathrm{N})$. In this two month study done at a tertiary care hospital, stool samples were processed and identified for Lactobacilli by standard protocols and colony counts recorded. Mann Whitney ' $U$ ' tests. 19 / 40 samples were positive for Lactobacilli in both groups. Colony counts in N: 1000-2000; O: 1500-2500. Lactobacilli counts though increased in the obese were statistically insignificant, implying as a genus, concentrations of Lactobacilli were marginally and insignificantly altered.

\section{Introduction}

Obesity is a disease prevalent in today's society, not only in high-income countries but also in middle and low income countries. It has led to over 2.8 million deaths annually worldwide. Over the years, the main factors that have been attributed to obesity are genetic predisposal and the lifestyle adopted by the individual. The significance of obesity lies in the fact that it leads to various complications, including the increased risk of atherosclerosis ${ }^{[1]}$.
In the year 2005, the link to obesity\& gut micro flora was established by Ley and Turnbaugh ${ }^{[2]}$. Their breakthrough has led to many studies world over along similar lines. Studies have led to the conclusion that the ratio of Bacteroides and Firmicutes in the gut micro flora is altered with obesity ${ }^{[3]}$. Lactobacilli, a sub classification of Firmicutes, have been dubbed as "good germs" ${ }^{44}$. This is mainly due to the capacity of Lactobacilli to generate an environment 
that stunts the capability of pathogenic organisms to colonize the gut and result in subsequent disease. This led to marketing and consumption of probiotic drinks and nutritional supplements

Some studies recently however, have concluded that obesity is linked to enrichment in the concentrations of gut Lactobacilli, particularly Lactobacilli reuteri $^{[5]}$. While the benefits of probiotics still remain debatable, the role of Lactobacilli in obesity is being studied as it has been proven that Lactobacilli act as growth promoters in livestock and impacts their weight gain ${ }^{[6]}$. It has traditionally been used in animal fodder to ensure optimal growth $^{[7]}$.

This culture based study aimed to establish a correlation between Lactobacilli and obesity, by its isolation, identification and estimation in subjects belonging to different BMI categories. We focused on concentrations of Lactobacilli as genus as opposed to particular species in this pilot study.

The main aim of this study includes, to establish a correlation between Lactobacilli present in the gut micro flora and obesity in a culture based study and thus:

a) Number of Lactobacilli in individuals with obesity

b) Substantiate or negate the role of gut micro flora in obesity

\section{Materials and Methods}

This study was a prospective cross-sectional one conducted for 2 months (from JulyAugust 2013) at a tertiary care teaching Hospital, Bangalore.

\section{Selection Criteria}

Inclusion Criteria: Subjects with obesity who were above 18 years of (WHO definition: $\mathrm{BMI}>30 \mathrm{Kg} / \mathrm{m}^{2}$ ) age group in the community. Control subjects with the BMI range $19-25 \mathrm{Kg} / \mathrm{m}^{2[8]}$.

Exclusion Criteria: BMI < $19 \mathrm{Kg} / \mathrm{m}^{2}$ for control group, BMI $<30 \mathrm{Kg} / \mathrm{m}^{2}$ for subjects with obesity group, Antibiotic administration < 1 month prior to sample collection, any recent disease or surgery involving the gastrointestinal tract.

\section{Study Outline}

The Study was conducted after obtaining ethical clearance from the Institutional Ethical Committee. The subjects were prescreened by the investigator for the criteria as indicated in the subject selection section. Only subjects who met these criteria with a signed informed consent were included in the study. A total of 40 subjects with 20 Obese and 20 Controls participated in this study.

\section{Sample Collection}

Clinical data (gender, date of birth, clinical history, weight, height and antibiotic use) were recorded using a standardized Performa. The samples were collected using sterile wide mouth plastic containers and were transported as soon as possible to the laboratory for analysis and the remaining refrigerated.

\section{Isolation of Lactobacilli}

Isolation of Lactobacilli from stool sample was done using a selective media called De Man Rogosa Agar (MRS Agar, Himedia, INDIA). After thawing to room temperature, $100 \mathrm{mg}$ of stool sample was mixed with $9 \mathrm{ml}$ of normal saline to get $1 / 10$ dilution. Then serial dilutions were made by taking $1 \mathrm{ml}$ aliquots and transferring to new 
tubes with $9 \mathrm{ml}$ saline solution. Finally $1 \mathrm{ml}$ from each tube with dilutions from 1/10 up to $1 / 10,000$ was inoculated using $1 \mathrm{ml}$ pipette onto MRS agar media [9]. (MRS media is a specific media proven to be highly sensitive for lactobacilli growth.) The plates were then incubated at $37^{0} \mathrm{C}$ for $48-$ 72 hours in anaerobic atmosphere (using candle jar) ${ }^{[10]}$.

After 48 hours, the numbers of colonies were counted from the plate having highest dilution from which the colonies were countable. In the present study the highest dilution taken was 1/1000.

\section{Identification of Lactobacilli}

The isolates were microscopically examined for Gram stain, motility was checked by hanging drop method, catalase test by using freshly prepared $3 \% \mathrm{H}_{2} \mathrm{O}_{2}{ }^{[11]}$, oxidase test by using tetra methyl-p- phenylene diamine dihydrochloride were done. Isolates showing gram positive bacilli which were non-motile, catalase and oxidase negative were considered as putative Lactobacillus and subjected to further biochemical tests. The biochemical tests performed were Indole, Methyl red (MR), Vogues Proskauer (VP), Simmon's Citrate test\& Nitrate reduction test.

Identification of isolates was based on Gram stain, morphology, growth characteristics on selective media such as MRS (HI-MEDIA, INDIA) agar and biochemical tests as recommended in the Bergey's Manual of Determinative Bacteriology ${ }^{[12]}$.

\section{Results and Discussion}

A total of 40 samples were collected over a period of two months. Of these samples, 20 belonged to the obese volunteer group and 20 belonged to the control group.
On tabulation of the age distribution of the two groups, most of the volunteers in the obese group belonged to the individuals aged from 30 to 50, which held true even for the volunteers who did not meet the exclusion criteria. (Table 1)

As per Figure 1, there were a higher number of co-morbidities associated with obesity, mainly, diabetes mellitus, and hypertension. In females, particularly, hypothyroidism was a cause of obesity.

\section{Analysis of the Colony Counts}

Control Group: The colony counts in the Control group was found to be fairly consistent and most of the colony counts fell in the range of 1000-2000 colonies per petridish of the diluted sample, as per Figure 2 . They showed very slight variation within the group

\section{Obese Group}

Figure 3, shows colony counts in the obese group, ranging from 400-4000 colonies per petridish. The average range of most of the colony counts was from 1500-2500. A clear increase in colony counts was seen in this obese group.

The colony counts were constant for a particular age group and showed consistent counts with few variations. In the middle age group and older age group, there was a decrease in the number of colony counts as opposed to the younger age group. In the obese group the middle aged group showed maximum variation and increase in overall counts.

The colony counts obtained in the female group revealed very few variations in the count. Whereas, a more obvious and pronounced variation was observed in the male group.3 samples reported a colony 
count of less than 1000 colonies per petri dish as per Figures 2 and 3.

Among the vegetarians in the control group, the colony counts were consistent whereas, it was highly variable in the obese group. Amongst the non-vegetarians, the colony counts in the two groups were in a similar range, barring one or two exceptions.

\section{Application of the Statistical Model - Mann Whitney $U$ test}

The Mann Whitney U test was applied to the two groups as a whole, and the value of $\mathrm{p}=$ 0.3192 ( $p<0.05$ is significant). The statistical model was applied to the various parameters as well with the values as follows:

In the present study females showed very few variations in colony counts. Males showed more variations in colony counts when compared to females, but it was found to be statistically insignificant as shown by the ' $p$ ' values.

Though, there were variations in the colony counts in vegetarians compared to nonvegetarians, it was statistically insignificant as shown in the above table.

This culture based study aimed to establish a correlation, if any, between Lactobacilli and obesity utilizing MRS agar, which was effective in isolating Lactobacilli.

The count of Lactobacilli were increased in obese volunteers, however, statistically it was found to be insignificant $(p=0.3192)$. This may have occurred as the range of colony counts varied between 1500-4000 colonies in the obese group. While some studies found a significant increase in the colony counts even with a smaller sample size ${ }^{[5]}$, some increased the sample size till they found significant alterations ${ }^{44}$.
This finding correlates with Study of Fabrice Armougum on bacterial communities of the gut micro biota which revealed a shift in the ratio of Firmicutes and Bacteroides in obese patients ${ }^{[13]}$. Also there was an increase of lactobacillus in some obese individuals and an increase of $M$. smithii in anorexic patients. Study of M. Million showed that there was a trend towards higher prevalence of lactobacillus in obese compared with lean patients. Occurrence of $L$. reuteri was more in obese patients when compared with lean patients $^{[5]}$.

When the data was analyzed in smaller pieces, considering the parameters of gender \&diet, variations did exist but; they were again found to be statistically insignificant. In terms of diet in females, most of the colony counts were in the same range and had only marginal variations. In males however, the variation in colony counts is more obvious and pronounced. Though there was more variation of colony counts in males when compared to females it was found to be statistically insignificant.

The study of Peiru Xu, revealed a significant decrease in the number of Bacteroides in the feces of obese Kazakh girls; no significant changes in Firmicutes numbers were noted. An association between reduced gut Bacteroides and Bact/firmicutes ratio obesity in female Kazakh children was identified. The above study also found that the difference inBacteroides levels observed between normal and obese children were mainly among the girls but not in boys ${ }^{[14]}$.

Another factor that needs to be accounted for is the level of fiber that the volunteer consumes. This translates into a change in the levels of various bacteria that are passed in the stool ${ }^{[15]}$. There were variations in the colony counts among vegetarians in the present study than non-vegetarians. 
Especially few obese vegetarians who consumed more dietary fibre had very lower counts of Lactobacilli. Our findings are in accordance with other studies wherein higher numbers of Bifidobacteria but significantly lower levels of anaerobic bacteria like Lactobacilli and lecithinase negative Clostridia were detected in rural elderly compared to urban elderly in Japan. This was due to lower intake of dietary fibre in the latter group.
The variation of the colony counts in particular age group is a pointer that age may also be a parameter in altering the colony counts significantly. In the obese middle aged group there was a maximum variation and increase in overall counts. Our findings are in contrast to earlier studies done on Asian populations which indicated a decrease in Bifidobacteria and an increase in Lactobacilli and Clostridia in elderly compared to adult subjects.

Table.1 Age Distribution in the Two Groups

\begin{tabular}{|c|c|c|}
\hline Age group & Obese Group & Control Group \\
\hline $18-19$ & 0 & 1 \\
\hline $20-29$ & 2 & 10 \\
\hline $30-39$ & 7 & 3 \\
\hline $40-49$ & 6 & 4 \\
\hline $50-59$ & 4 & 2 \\
\hline$>60$ & 1 & 0 \\
\hline
\end{tabular}

Table.2 Mann Whitney U Test Results for Various Parameters

\begin{tabular}{|c|c|c|}
\hline \multicolumn{2}{|c|}{} & \multicolumn{2}{c|}{ P values } \\
\hline \multirow{2}{*}{ Gender } & Male & 0.3 \\
\cline { 2 - 3 } & Female & 0.14 \\
\hline \multirow{2}{*}{ Diet } & Non vegetarians & 0.17 \\
\cline { 2 - 3 } & Vegetarians & 0.32 \\
\hline
\end{tabular}

Figure.1 Morbidity Profile of the Two Groups

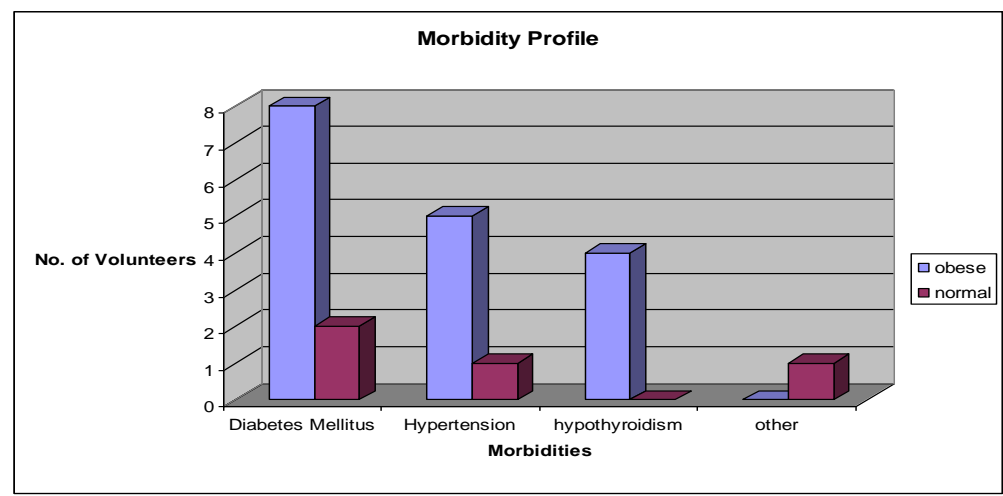


Figure.2 Total Colony Counts in the Control Group

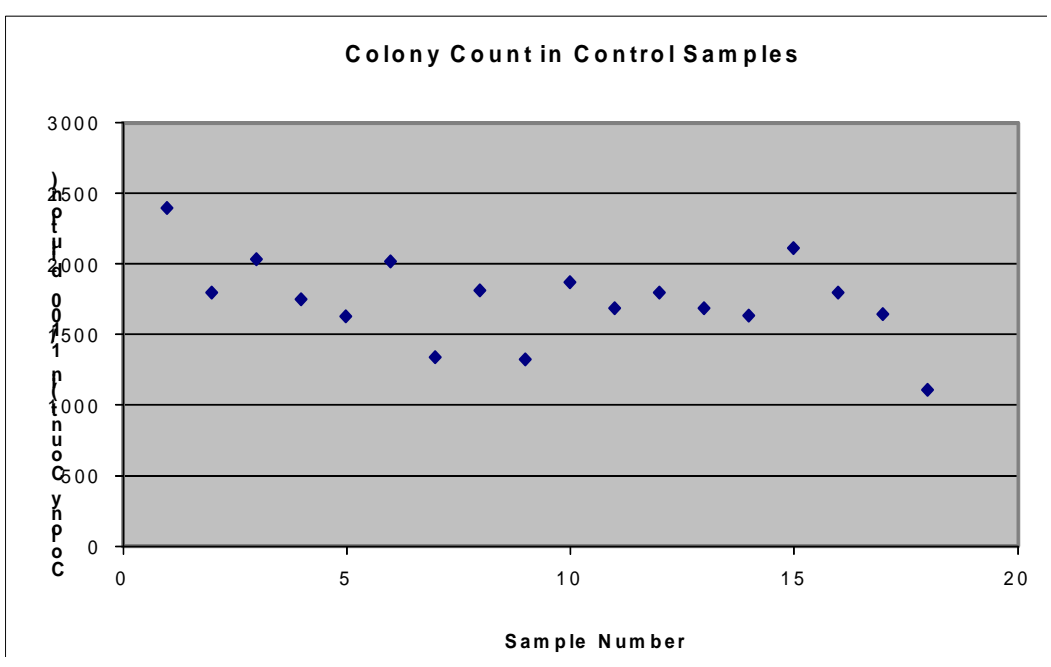

Figure.3 Total Colony Counts in the Obese Group

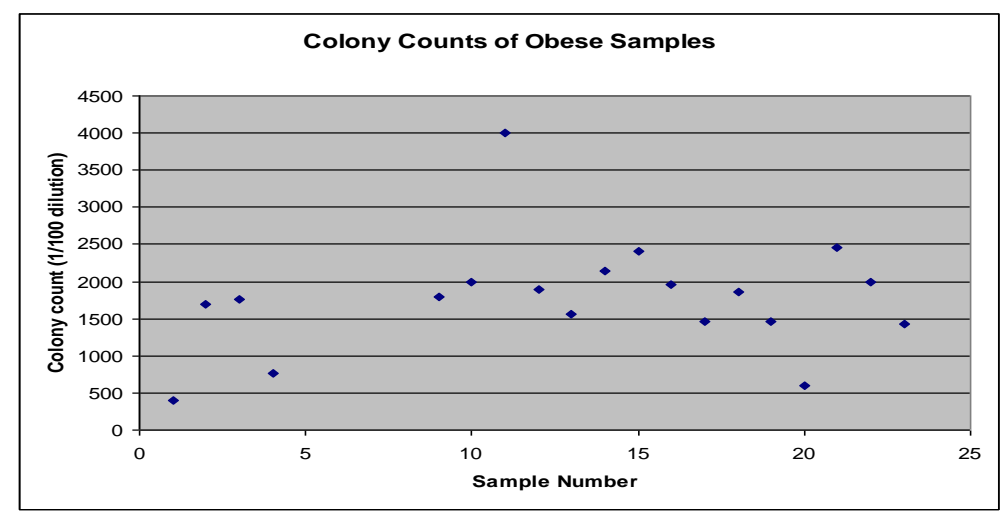

Co-morbidities associated with the obese group gave consistent colony counts as well, implying that the disease maintained physiological balance in the individuals or affected the gut micro flora uniformly. In fact, probiotic dahi for therapeutic administration in Diabetes Mellitus has been looked into. A probiotic dahi-supplemented diet was found to have significantly delayed the onset of glucose intolerance, hyperglycemia, hyperinsulinemia, dyslipidemia, and oxidative stress in high fructose-induced diabetic rats, indicating a lower risk of diabetes and its complications [16].

The relation and mechanism of Lactobacilli and obesity is contradictory. While it has been used as fodder in livestock to increase mass ${ }^{[8]}$ it has also been attributed to deconjugating bile acids and therefore, limiting fat absorption. The mechanisms or pathways involved in contribution to obesity are: 1 . Increasing energy recovery from the diet. 2. Modulating liver lipogenesis. 3. Regulating appetite through gut satiety hormones. 4. Activating innate immunity through LPS - Toll like receptors. The first pathway that influences host energy storage is related to energy extraction from undigested food components. Nutrients which escape the digestion, due to host's limited capability of glycoside hydrolases to 
digest complex dietary plant polysaccharides are fermented by gut microbes into monosaccharides and short-chain fatty acids (SCFAs), such as acetate, propionate, and butyrate representing an important energy source for the body [17]. Since these pathways have all been linked to the gut microbial community, dietary manipulation of this "organ system" has the potential to reduce risk for obesity.

In conclusion, there was an increase in the overall levels of the genus Lactobacilli in the obese volunteers but this was found to be statistically insignificant. Taking sex and diet into consideration as well, a similar result was obtained. This result however is not the final say in the matter as many other factors need to be taken into consideration such as co-morbidities and age. Further studies, however, can aid in the therapeutic management of obesity.

\section{Limitations of the Study}

The present study had a small sample size and concentrated on the genus as a whole, but it requires further work like differentiating the species of Lactobacilli and quantifying each of them. A better comparison then can be made if there is alteration in the levels of species.

\section{Recommendation}

The study design can be changed to include larger sample size with subjects of a specific gender, diet etc. By fixing parameters therefore, the study can be more specific.

Speciation of the Lactobacilli obtained in the samples can shed more light into the alteration in the concentration of particular strains of Lactobacilli.
Also, to ensure sensitivity, qPCR may be used.

\section{Acknowledgement}

The authors would like to acknowledge the STS Program of ICMR under which this research was carried out.

\section{References}

1. World health organization Obesity and overweight Fact sheet May 2012

2. Ley RE et al 2005. Obesity alters gut microbial ecology. Proc Natl Acad Sci USA.; 102: 11070-75.

3. Turnbaugh PJ et al 2006. An obesityassociated gut microbiome with increased capacity for energy harvest Nature.;444(7122):1027-31

4. Gabriela Sinkiewicz. 2010. Lactobacillus reuteri in health and disease, Malmö University, Health and Society Doctoral Dissertations:3

5. Million M, Maraninchi M, Henry M 2012. Obesity-associated gut microbiota is enriched in Lactobacillus reuteri and depleted in Bifidobacterium animalis and Methanobrevibacter smithii, Int $\mathrm{J}$ Obes (Lond). June; 36(6):817-825.

6. Reid G1999. The Scientific Basis for Probiotic Strains of Lactobacillus; Appl Environ Microbiol.; 65(9): 3763-3766

7. Dunham HJ et al 1998. Avian growth depression in chickens induced by environmental, microbiological, or nutritional stress is moderated by probiotic administrations of Lactobacillusreuteri. Biosc Microflor.;17:133-139

8. WHO Expert Consultation 2004. Appropriate body-mass index for Asian populations and its implications for policy and intervention strategies. Lancet.; 363(9403):157-63. 
9. John Lindquist 2012; Dilution Plating http://www.jlindquist.net/generalmicro/ 102dil1.htm

10. JacksonMS, Bird AR, McOristAL (2002).Comparison of two selective media for the detection and enumeration of Lactobacilli in human faeces. Journal of Microbiological Methods 51,313-321

11. Pascual LM, Daniele MB, Pa jaro C, Barberis LT 2006. Lactobacillus species isolated from the vagina: identification, hydrogen peroxide production and nonoxynol-9 resistance Contraception. Jan; 73(1):78-81.

12. Holt JG, Kreig NR, Sneath PHA, Stanley JT, Williams ST 1994. Regular, nonsporing gram positive rods in Bergey's Manual of determinative Bacteriology, $9^{\text {th }}$ Ed. Lippincott Williams\&Wilkins, New York; 565-67.

13. Armougom F, Henry M, Vialettes B, Raccah D, Raoult D 2009. Monitoring bacterial community of human gut microbiota reveals an increase in Lactobacillus in obese patients and Methanogens in anorexic patients. PLoS One. ; 4(9):e7125. doi: 10.1371/journal.pone.0007125.

14. Xu P, Li M, Zhang J, Zhang T 2012. Correlation of intestinal microbiota with overweight and obesity in Kazakh school children. BMC Microbiology; 12:283.

15. Hooda S, et al 2012. 454 Pyrosequencing Reveals a Shift in Fecal Microbiota of Healthy Adult Men Consuming Polydextrose or Soluble Corn Fiber. Journal of Nutrition,; 142 (7): 1259

16. Yadav H, Jain S, Sinha PR 2007. Antidiabetic effect of probiotic dahi containing Lactobacillus acidophilus and Lactobacillus casei in high fructose fed rats. Nutrition. Jan; 23(1):62-8.

17. Shimada K, Bricknell KS, Finegold SM 1969. Deconjugation of bile acids by intestinal bacteria: review of literature and additional studies. $\mathbf{J}$ Infect Dis.;119:73-81

\section{How to cite this article:}

Akshita Ramani, S. Sangeetha and Y. K. Harshika. 2016. Lactobacilli and Obesity. Int.J.Curr.Microbiol.App.Sci. 5(2): 711-718. doi: http://dx.doi.org/10.20546/ijcmas.2016.502.079 